\title{
Role of the WASP and WAVE family proteins in breast cancer invasion and metastasis
}

\author{
This article was published in the following Dove Press journal: \\ Breast Cancer: Targets and Therapy \\ 24 April 2015 \\ Number of times this article has been viewed
}

\author{
Bethan Frugtniet \\ Wen $G$ Jiang \\ Tracey A Martin \\ Cardiff-China Medical Research \\ Collaborative, Cardiff University \\ School of Medicine, Cardiff University, \\ Cardiff, UK
}

\begin{abstract}
The Wiskott-Aldrich syndrome protein (WASP) and WASP family verprolinhomologous protein (WAVE) family are a group of molecules that form a key link between GTPases and the actin cytoskeleton. The role of WASP/WAVE family proteins in the control of actin polymerization through activation of the actin-related protein $2 / 3$ complex is critical in the formation of the actin-based membrane protrusions seen in cell migration and invasion. For this reason, the activity of the WASP/WAVE family in cancer cell invasion and migration has been of great interest in recent years. Many reports have highlighted the potential of targeting the WASP/WAVE family as a therapy for the prevention of cancer progression, in particular breast cancer. This review focuses on the role of the WASP/WAVE family in breast cancer cell invasion and migration and how this relates to the molecular mechanisms of WASP/WAVE activity, their exact contributions to the stages of cancer progression, and how this can lead to the development of anticancer drugs that target the WASP/WAVE family and related pathways.
\end{abstract}

Keywords: WASP, WAVE, breast cancer, migration, invasion

\section{Introduction}

In females, breast cancer is the most frequently diagnosed malignancy and one of the main causes of cancer death, ${ }^{1}$ with survival rates much lower in patients with metastatic cancer. ${ }^{2}$ Metastasis, the process whereby cancer cells travel to and colonize distant sites through the lymphatic system or bloodstream, can signal a poor prognosis in patients. The metastatic cascade involves many complex cellular interactions and pathways. Invasion is the initial critical step in metastasis and describes changes in cell adhesion, degradation of the cell surroundings, and importantly, the motility acquired by the tumor cell that confers a migratory phenotype and allows the cell to travel through tissues. ${ }^{3}$ Briefly, the cellular processes that occur in a motile cell include polarization and extensions of actin-based protrusions, such as thin, finger-like filopodia and broader, sheet-like lamellipodia, in the direction of migration (Figure 1A). Adhesions between the actin cytoskeleton of a migrating cell and the extracellular matrix (ECM) or neighboring cells stabilize protrusions and form traction sites to allow the cell to move over them. ${ }^{4}$ Specialized actin-rich protrusions, such as invadopodia, can also be formed by cancer cells, allowing them to degrade the ECM and invade into the surroundings. ${ }^{5}$ Formation of the protrusions at the leading edges of the migrating cell is controlled by actin polymerization and regulators of the actin cytoskeleton. ${ }^{6,7}$ The Rho GTPase protein family members, Rho, Rac, and Cdc42 in particular, play a key role in coordinating the processes involved in cell migration and invasion through the control of actin dynamics. ${ }^{8}$ The Wiskott-Aldrich syndrome (WAS) protein (WASP) and WASP 


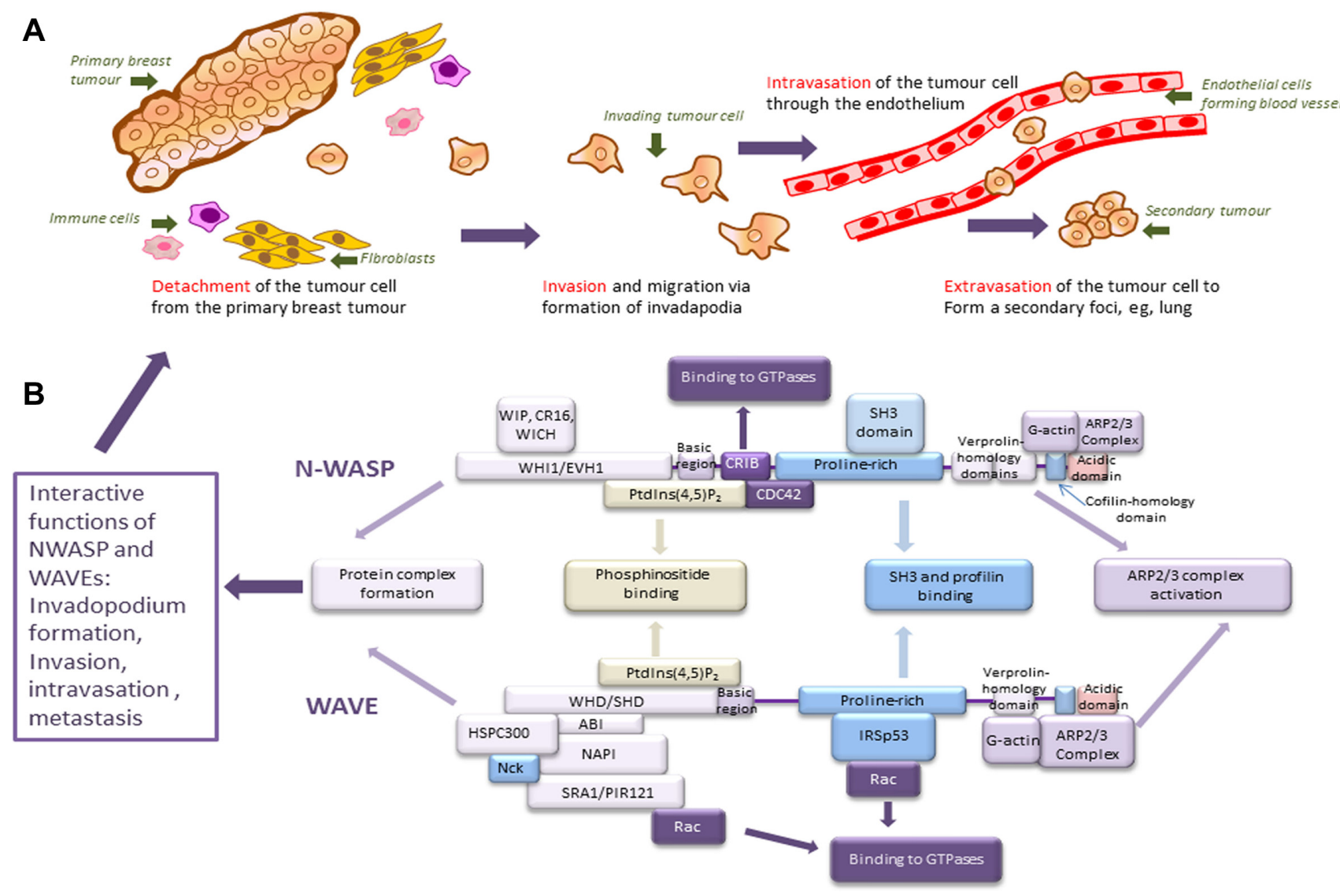

Figure I Structure and functions of WASP/WAVE family proteins.

Notes: (A) Metastatic cascade showing the role of migration and invadopodia in successful invasion, intravasation, and extravasation of the tumor cell from the primary breast tumor to secondary foci, eg, the lung. (B) The structure and functional motifs for nWASP and WAVE and the interactions between them. They are related to the functions of both these proteins in the control of the actin cytoskeleton and hence the migratory capacity of cells.

family verprolin-homologous protein (WAVE) family are a group of molecules that form a key link between GTPases and the actin cytoskeleton. ${ }^{9}$ The role of WASP/WAVE family proteins in the control of actin polymerization through activation of the actin-related protein (Arp)2/3 complex is critical in the formation of the actin-based membrane protrusions seen in cell migration and invasion. ${ }^{10}$ For this reason, the activity of the WASP/WAVE family in cancer cell invasion and migration has been of great interest in recent years. This is the main focus of this review, with an emphasis on WASP/ WAVE activity in breast cancer cell motility.

\section{WASPs and WAVEs Discovery}

In humans, the WASP/WAVE family is divided into two main subfamilies, ie, the WASP subfamily (referred to as WASPs) and WAVE subfamily (referred to as WAVEs). The first member of the WASPs to be identified was WASP itself, mutations of which were found to be the cause of WAS. ${ }^{11}$ WAS is an X-linked recessive disorder initially described in patients suffering with thrombocytopenia, eczema, and immunodeficiency, ${ }^{12}$ now commonly labeled the clinical triad of WAS symptoms. Patients exhibit varied severity of symptoms, with some suffering the full classic triad of clinical manifestations and often not surviving until adulthood, whereas others demonstrate a milder phenotype and have an improved survival rate. ${ }^{13}$ Different mutations of the WASP gene have been linked to the varied phenotypes of WAS, with evidence that mutations disrupting the activity of the important functional domains lead to more severe phenotypes. ${ }^{14,15}$

\section{Family members}

A molecule identified in the brain, but widely expressed in different tissues, which shares several functional motifs with WASP, was named neural WASP (nWASP) and is the second member of the WASP subfamily. ${ }^{16}$ WAVE1 (also referred to as Scar1, suppressor of cAR1) was first identified in 1998 as a regulator of the actin cytoskeleton through interactions with 
Arp2/3 downstream of Rac. ${ }^{17,18}$ Following identification of WAVE1, two further WAVE homologs, which were also found to associate with the Arp2/3 complex, were characterized and named WAVE2 and WAVE3. ${ }^{19}$ Novel members of the WASP/WAVE family have recently been identified based on the resemblance between characteristic domains in their protein structure. These are WASP and SCAR homolog, WASP homolog associated with actin, membranes, and microtubules, and junction mediating and regulatory protein. ${ }^{20-22}$

\section{Structure}

nWASP shares several multifunctional domains with WASP, including the WASP homology domain, GTPase-binding domain/Cdc42, and Rac interactive binding (GBD/CRIB) domain, a proline-rich region and VCA domain consisting of a verprolin-homology (V) region, a cofilin-like (C) region, and an acidic (A) region (Figure 1B, adapted from Takenawa and Suetsugu ${ }^{9}$ ). The WAVE family consists of WAVE1, WAVE2, and WAVE3, all of which have a WAVE homology domain but lack the GBD/CRIB domain and, like the WASPs, have a basic region, a proline-rich region, and a VCA domain. ${ }^{23}$ Endogenous WAVEs assemble into a heterologous, multimolecular complex referred to as the WAVE complex. The multiprotein complex associated with WAVE1 was first described as comprising several components, namely, p53-inducible messenger RNA (also called Rac2 associated protein/Sra1), NCK-associated protein, hematopoietic stem/ progenitor cell protein 300 , and $\mathrm{Abl}$ interactor $2 .{ }^{24}$ Complexes consisting of the same proteins, or their paralogs, as described for the WAVE1 complex were later identified for WAVE2 and WAVE3 as well. ${ }^{25-27}$

\section{Activation of the WASP/WAVE family}

The WASP/WAVE family regulates actin dynamics through interactions with many other factors, in particular, the Arp $2 / 3$ complex. Arp2/3 becomes activated when bound to the CA region on the C-terminal of WASP/WAVE proteins and initiates actin polymerization when bound in conjunction with an actin monomer binding to the $\mathrm{V}$ region. ${ }^{18,23,28,29}$ This interaction is the most important in terms of WASP function, but many other binding partners regulate WASP and WAVE activation.

Under resting conditions, nWASP and WASP exist in an autoinhibited, folded conformation where an interaction between the GBD domain in the N-terminal region and the C-terminus masks the VCA region. This interaction inhibits access of the Arp2/3 complex to the CA region and so WASP and nWASP remain inactive. ${ }^{30,31}$ The competitive binding of ligands such as Rho GTPase, Cdc42, and phosphatidylinositol 4,5-bisphosphate (PIP2) with WASPs can disrupt the interactions between the $\mathrm{C}$-termini and $\mathrm{N}$-termini that mask the VCA region. Cdc42 binds to GBD and PIP2 can interact with the basic region. Either of these interactions can destabilize the autoinhibited conformation of WASPs and enhance the binding of the other ligand, leading to exposure of the VCA region and activation of WASP family proteins. $^{32,33}$

The mechanism of activation of WAVEs has been the subject of debate for many years. It is now clear that the native form of WAVEs, in a multiprotein complex, is inactive with inhibited actin polymerization ability. ${ }^{34}$ This has been confirmed by studies examining the reconstitution of the WAVE complex and purified native WAVE complexes. These independent reports also suggest that WAVEs are inhibited in a similar fashion to WASPs, ie, through masking of the VCA region by interactions in the WAVE complex. ${ }^{35,36}$ Since WAVEs do not possess a GBD like WASPs, they do not directly interact with Rho-GTPases; however, they do act as downstream effectors of the Rho-GTPase Rac, as well as other signals. ${ }^{17}$ A conformational change brought about by Rac binding to the Sra component of the WAVE complex seems to release the VCA region. ${ }^{35}$ Phosphatidylinositol (3,4,5)-trisphosphate (PIP3) has also been implicated in the mechanism of activation of WAVEs. It has been shown that PIP3 recruits the WAVE complex to the plasma membrane through binding to the basic domain of WAVE2 and acts in synergy with IRSp53 (also called brain-specific angiogenesis inhibitor 1-associated protein 2) to activate WAVE2 through Rac activity. ${ }^{37,38}$ For comprehensive information on the mechanisms of activation of the WASP/WAVE family, see reviews that have covered this in more detail.9,39

\section{WASPs, WAVEs, and cell motility}

When members of the WASP/WAVE family are active, the VCA region is exposed, allowing the Arp2/3 complex and an actin monomer to bind to it, leading to nucleation of a new actin filament. Activation of WASP/WAVE family members has been implicated in many biological processes where reorganization of the actin cytoskeleton is required, such as vesicle trafficking, microbial infection, and cell-substrate adhesion (see reviews by Takenawa and Suetsugu ${ }^{9}$ and Takenawa and $\mathrm{Miki}^{23}$ ). The main focus of this review is their role in the control of cell motility, with a particular focus on cancer cell motility and metastasis. 


\section{WASPs, WAVEs, and cell protrusions}

The motility of eukaryotic cells is accomplished by dynamic remodeling of the actin cytoskeleton. Cell motility in twodimensional environments, such as on culture dishes or over the ECM, is driven by formation of lamellipodia and filopodia at the leading edge. It is traditionally believed that lamellipodia are the key structures that provide the main driving force in two-dimensional cell motility. This was first demonstrated by a simple experiment where a section excised from a fish keratinocyte lamellipodium was seen migrating at a speed similar to that of an intact cell. ${ }^{40}$ Filopodia, although they are not implicated as being the major force behind cell motility, seem to have a role in sensing the environment outside the cell and guide cell migration and forming of adhesions with the ECM. ${ }^{41,42}$ The organization of actin filaments is different in the various protrusions that drive cell motility. Actin filaments assemble in a branching network in lamellipodia, whereas in filopodia they form long parallel bundles. ${ }^{43} \mathrm{WASP} / \mathrm{WAVE}$ family proteins interact with the Arp2/3 complex to induce formation of branched actin networks, where actin filaments are assembled at a $70^{\circ}$ angle from the sides of pre-existing filaments, which are the main structures responsible for formation of lamellipodia. ${ }^{44}$

Although the main mode of cancer cell migration is through three-dimensional matrices, which are discussed later in this review, many advances in our understanding of the mechanics of cell motility and the role of WASP/WAVE family proteins have been as a result of studying features of cells that move in two dimensions. Some of these findings are briefly discussed here.

Initial work investigating the role of WASP/WAVE family proteins in cell motility found that $\mathrm{Cdc} 42$ signaling requires nWASP activity in order to form the actin structures seen in filopodia, and WAVE1 activity downstream of Rac is required for formation of lamellipodia. ${ }^{17,31}$ However, it is now clear that these studies give a limited understanding of the role of the WASP/WAVE family, as nWASP activity is not essential for the formation of filopodia in response to $\mathrm{Cdc} 42$ signaling in fibroblasts. ${ }^{45}$ Moreover, many reports have implicated nWASP in the control of the actin dynamics in lamellipodia through demonstrating nWASP activity localized at the leading edge of lamellipodia in a variety of cell types. ${ }^{46-48}$ However, several studies have also shown that depletion of WASP or nWASP activity does not affect the formation of lamellipodia in several cell types, including mice fibroblasts, HeLa cells, and Drosophila S2 cells. ${ }^{49-51}$ Instead, many reports support the idea that members of the WAVE subfamily are key in the control of lamellipodia formation in various cell types. ${ }^{50-53}$
In particular, induced WAVE2 deficiency in mouse endothelial, embryonic fibroblast, melanoma, and macrophage cells resulted in impaired ability of the cells to form lamellipodia and a decrease in cell motility. ${ }^{53-56}$ These findings collectively suggest that the WAVE subfamily, particularly WAVE2, has a more important role in the formation of lamellipodia protrusions and mesenchymal migration, and hence some aspects of cell motility, than the WASP subfamily, but that there is evidence that the contribution of the WASP/WAVE family members to the control of actin dynamics varies in different cell types. Most of these studies analyzing the generation of filopodia and lamellipodia have used cells cultured on rigid two-dimensional substrates which can help us understand the mechanisms involved in cell motility. While formation of these types of cell structures does take place in motile cells in vitro, cells in vivo, and especially cancer cells, generally use different methods of migration as they have to invade through a three-dimensional matrix. ${ }^{57}$ Nevertheless, cancer cells do still utilize actin-based protrusions to allow them to invade through the ECM and migrate during the process of metastasis. The WASP/WAVE family proteins have been shown to be important in the formation of these protrusions, which are more specific to motility in three dimensions and in migration of cancer cells, as well as in two-dimensional motility. Interestingly, a recent report has suggested that the WASP/WAVE family members may have different roles depending on the mode of cellular migration. ${ }^{58}$ This group targeted the WAVE complex using small interfering RNA (siRNA) silencing techniques and found that although it promotes motility in two dimensions, disruption of the WAVE complex promotes cell invasion and enhances focal adhesion kinase activity and nWASP localization at invasive protrusion sites. They also proposed that the WAVE complex and nWASP have opposing roles in three-dimensional epithelial cell invasion and that there may be an interplay between WASP/WAVE family members in the control of various modes of cell motility. Reports that examine the role of the WASP/WAVE family in three-dimensional motility, and in particular the motile behavior of cancer cells, are the main focus of the rest of this review.

\section{WASPs, WAVEs, and cancer cell protrusions}

Podosomes and invadopodia are specialized membranesurface structures that have a role in the migration of invasive cells and degradation of the ECM. Podosomes are formed in various cell types, including monocytic cell lineages, smooth muscle cells, and endothelial cells, whereas invadopodia 
usually refer to protrusions similar in structure and function to podosomes but are found in invasive cancer cells. Invadopodia contain a core of actin filaments, as well as adhesion proteins, proteinases for ECM degradation, and many signaling molecules. ${ }^{5,59}$

\section{nWASP and invadopodia}

nWASP and the Arp2/3 complex have been implicated in the formation of invadopodia. Using a biosensor, nWASP has been shown to be active at invadopodia in MTLn3 rat mammary adenocarcinoma cells. ${ }^{46}$ nWASP staining has also shown clear localization with invadopodia in these highly metastatic cells, and also other cancer cell types. Furthermore, RNA interference techniques have been used to show that nWASP and some of its interaction partners, in particular the Arp2/3 complex, are crucial for formation of invadopodia. WAVE1 and WAVE2 showed no invadopodiumspecific staining and were not shown to be essential for their formation. ${ }^{60}$ Such findings agree with other studies in their conclusion that nWASP is involved in formation of invadopodia in various cell types, including rat fibroblasts and adenocarcinoma cells. ${ }^{61,62}$

More recently, a study has proposed that nWASPmediated formation of invadopodia is essential in breast cancer invasion, intravasation, and metastasis to the lungs. This research group induced MTLn3 cells to overexpress the dominant negative form of nWASP, which lacks the ability to activate the Arp $2 / 3$ complex and so competitively inhibits endogenous nWASP, and reduced nWASP protein expression in these cells by $75 \%$ using short-hairpin RNA (shRNA) silencing techniques. Interference with endogenous nWASP activity in these cells significantly reduced formation of invadopodia and their proteolytic activity when cultured on a gelatin matrix. ${ }^{63}$

Since formation of invadopodia correlates with the invasive capacity of cancer cells, it is clear that the nWASP pathway could be considered a therapeutic target for inhibition of invasion in various cancers, in particular breast cancer, as demonstrated by use of mammary adenocarcinoma cells in the mentioned studies. ${ }^{64}$

\section{WASPs, WAVEs, and cancer}

Following investigation of WASP/WAVE family proteins in control of cellular motility and formation of cellular projections in cancer cells, there has been a large focus on the role of WASP/WAVE family proteins in cancer cells and tissues. Several studies mentioned here have explored WASP/WAVE proteins in various types of cancers.

\section{WAVEs and melanoma}

A study focused on WAVE subfamily activity in invasion and metastasis found higher levels of WAVE1 and WAVE2 protein and Rac activity in highly invasive B16F10 mouse melanoma cells compared with non-metastatic B16 parental cells. ${ }^{55}$ RNA interference techniques were used to silence WAVE1 and WAVE2 in these malignant cells, and membrane ruffling, Rac-induced invasion, motility, and metastasis were found to be suppressed in response to WAVE2 knockdown, whereas WAVE1 knockdown appeared to have little effect on these cellular behaviors. Further, WAVE2 appeared to be concentrated at the edges of the protrusions in migrating cells. Also, this study undertook in vivo studies to examine the effect of WAVE inhibition on metastasis. Cells with suppressed WAVE2 activity, through RNA interference again, suppressed metastasis of B16F10 cells to the lungs when injected intravenously into female $\mathrm{C} 57 \mathrm{BL} / 6$ mice, whereas WAVE1 RNA interference did not prevent colonization of the lungs by B16F10 cells. This study suggested that WAVE2 is necessary for motility of B16F10 cells and that WAVE2 suppression may be an effective means to block invasion and metastasis. ${ }^{55}$

\section{WAVE2 as a prognostic indicator}

The value of using WAVE2 as a prognostic indicator for various cancers has been explored by many studies. WAVE2 expression has been examined in 112 hepatocellular carcinoma tissues through immunohistochemistry, real-time polymerase chain reaction (RT-PCR), and Western blotting experiments to determine if any correlation exists between expression and prognosis. WAVE2 expression was found to be elevated in hepatocellular carcinoma tissues and, importantly, this correlated significantly with a poor prognosis of hepatocellular carcinoma. ${ }^{65}$ Further, coexpression of Arp2 and WAVE2 was studied by immunohistochemical staining on sections of 115 adenocarcinomas of the lung from different classes. Arp2 and WAVE2 were coexpressed in a significantly higher proportion of cancer cells from patients with lymph node metastasis but at a significantly lower level in bronchioloalveolar carcinomas, which have a less malignant character. Arp2 and WAVE2 coexpression was also correlated with a poorer patient outcome, leading to a suggestion that coexpression of Arp2 and WAVE2 may be a phenotype of tumor cells that acquire invasive potential and that they have a role in the mechanism of cancer metastasis. ${ }^{66}$ This finding was supported by another study that examined the spatial expression of Arp2 and WAVE2 using immunohistochemistry in 154 colorectal cancer samples. Both Arp2 and WAVE2 
expression was detected in $35.7 \%$ of the cancer cells in the cohort but no colocalization was detected in normal colonic epithelial cells. Their comparison of expression levels and patient outcome determined that colocalization of Arp2 and WAVE2 was significantly predictive of liver metastasis in colorectal carcinoma. ${ }^{67}$

\section{WAVEI and WAVE3 in prostate cancer}

Fernando et al investigated the expression of WAVE 1 and WAVE3 in prostate cancer cells and their role in metastasis and invasion. ${ }^{68,69}$ Immunohistochemical staining of prostate cancer tissue showed that there was higher staining intensity of WAVE1 and WAVE3 compared with normal prostate epithelial cells. Knockdown of WAVE1 and WAVE3 was carried out using hammerhead ribozyme transgenes. These were transfected by electroporation into metastatic prostate cancer cell lines, DU-145 and PC-3, both of which showed high expression levels of WAVE1 and WAVE3 through RT-PCR prior to transfection. In vitro invasion and growth assays were used to study the impact of WAVE1 and WAVE3 knockdown on cell behavior. Loss of endogenous WAVE1 and WAVE3 resulted in a significant reduction in the invasive ability of both prostate cancer cell types. Additionally, in both DU-145 and PC-3 cells, knockdown of WAVE1 and WAVE3 achieved a significant decrease in the growth rate after 120 hours when compared with wild-type cells. ${ }^{68,69}$

An independent group also carried out a study to determine the effect of WAVE3 (labeled WAS protein family member 3 in that study) in prostate cancer metastasis, ${ }^{70}$ which supports many of the findings reported by Fernando et al. ${ }^{68}$ High expression levels of WAVE3 were observed in metastatic PC-3 and DU-145 prostate cancer lines through quantitative RT-PCR, and immunohistochemical analysis of high-grade human prostate cancer demonstrated increased levels of WAVE3 when compared with normal prostate epithelium. Knockdown of WAVE3 was achieved using shRNA methods and a significant decrease in cell motility and invasion in vitro was seen through scratch wounding assays, which were only carried out using the DU-145 cell model, and Matrigel invasion assays. Further, knockdown of WAVE3 expression reduced the soft agar colony-forming ability of prostate cancer cells, suggesting that WAVE3 might also have a role in establishment of tumors. The effect of WAVE3 knockdown on in vivo tumor growth and metastasis of prostate cancer cells was observed by injecting the knockdown models into the flanks of severe combined immunodeficiency disease (SCID) mice. Tumor growth rate and tumor weight following a 3-week period were significantly decreased with less or no evidence of metastasis to the lungs when WAVE3 knockdown DU-145 and PC-3 cells, respectively, were implanted into SCID mice when compared with the control group. ${ }^{70}$ These findings demonstrate that WAVE3 may have a role in promoting motility, invasion and metastasis in prostate cancer cells and that a loss of WAVE3 function affects tumor development, so highlighting the importance of WAVE3 as a research target.

\section{WASPs, WAVEs, and breast cancer}

As described, WASP/WAVE protein activity is not just important in the motility of healthy cells but has also been suggested to have a role in various types of cancer. A large number of studies have focused on breast cancer. Numerous reports have identified WASP/WAVE family members in the control of the motile behavior of breast cancer cells and also as a prognostic indicator of breast cancer progression in patients. Some of the relevant findings are detailed below.

\section{WASP and breast cancer}

A recent study has identified that WASP has a role in regulating leukocyte-dependent breast cancer metastasis. ${ }^{71}$ This research group built on previous studies showing that the interaction between epidermal growth factor-secreting macrophages and colony-stimulating factor 1 -secreting breast cancer carcinoma cells promotes invasion. ${ }^{72,73}$ They hypothesized that WASP deficiency may block the interaction between tumor-associated macrophages and breast carcinoma cells, and hence tumor progression toward invasion since chemotaxis of macrophages toward colony-stimulating factor 1 in patients with WAS, who have defective WASP activity, is abolished. ${ }^{74}$ The study showed that in order for macrophages derived from bone marrow and MTLn3 breast cancer carcinoma cells to adopt migratory phenotypes and coinvade a three-dimensional collagen matrix, WASP activity in the macrophages is required. Further, injection of fluorescent MTLn3 cells into the mammary glands of WASP-/- and WASP+/- mice found no difference in initial tumor growth. However, metastasis to the lung was significantly reduced in mice that lacked WASP activity, a finding that was supported by studies in highly metastatic human breast cancer MDA-MB-231 cells and a transgenic model of mammary carcinogenesis. This suggested that leukocyte-restricted WASP expression is required for metastasis of breast tumor cells to the lungs. Overall, this study indicates a role of WASP signaling in motility, invasion, and intravasation, and hence metastasis, of breast cancer tumor cells possibly by regulation of epidermal growth factor shedding from tumor-associated macrophages. ${ }^{71}$ 


\section{nWASP and breast cancer}

nWASP has been implicated in the progression of mammary tumors by one group of researchers who interfered with endogenous nWASP levels in MTLn3 cells using shRNA techniques. When these cells were injected into the mammary glands of SCID mice and rats, a significant reduction in the number of circulating tumor cells and lung metastases was observed when compared with the controls. Further, in vivo time-lapse images of control and nWASP-deficient GFP-labeled cells in tumors demonstrated that there was a dramatic decrease in motile cells and formation of invadopodium-like protrusions in nWASPdeficient tumors compared with control tumors. This study demonstrates that nWASP activity is involved in proteolytic protrusion formation, and consequently the invasion and metastasis to the lungs of MTLn3 cell tumors..$^{63} \mathrm{~A}$ further study treated MDA-MB-231 breast cancer cells with the nWASP inhibitor, ${ }^{75}$ wiskostatin, which acts to stabilize the autoinhibited state of nWASP. ${ }^{76}$ A resulting decrease in motility of cells treated with the inhibitor was detected using electric cell-substrate impedance sensing, a method that detects the movement of cells over an electrode in real time. This study suggested that interactions between nWASP and the tight junction component, claudin-5, shown by immunoprecipitation and immunoblotting, may be involved in the motility of cancer cells. ${ }^{75}$

Interestingly, another study using immunohistochemical and quantitative RT-PCR analysis of human breast tissues has indicated that expression of nWASP in breast tumors is significantly lower than in normal background mammary tissues, and reduced expression of nWASP seems to be associated with a poor prognosis for patients. Significantly lower levels $(P<0.05)$ were seen in patients who developed metastatic disease or died from breast cancer. Further, induced overexpression of nWASP in MDA-MB-231 cells significantly reduced their invasiveness, motility, and growth, but showed a more adhesive phenotype. ${ }^{77}$

Although these studies suggest an opposing role of nWASP in the control of breast cancer cell motility, they both recognize that nWASP may be related to the behavior of breast cancer cells and progression of tumor formation. The molecular mechanisms responsible for the changes in breast cancer cell motility, invasion, and migration related to nWASP activity are still being explored, but several interaction partners and activators of nWASP have already been implicated, such as Cdc42-interacting protein 4 and focal adhesion kinase, although it should be noted that these can work independently of the nWASP pathway. ${ }^{78,79}$

\section{WAVE2 and breast cancer}

The association of WAVEs with breast cancer has been examined in a study of the expression of each family member at the mRNA and protein levels in 122 human breast cancer samples and 32 normal breast tissue samples. The levels of expression were also analyzed for any correlation with pathological and clinical outcome. Differential expression of the WAVE subfamily members in breast cancer tissues was shown in this study, and the main significant finding was that of high levels of WAVE2 in patients who subsequently died of breast cancer within a 10 -year follow-up period. ${ }^{80}$

Several studies have highlighted the potential therapeutic value of targeting the WASP/WAVE family or molecules up/downstream of them in their respective signaling pathways in the inhibition of cancer. Two research groups have recently investigated rhapontigenin and pterostilbene as potential inhibitors of breast cancer cell metastasis that may act through the WAVE2 pathway. ${ }^{81,82}$ Both studies showed that application of each treatment can suppress the migratory and invasive activity of MDA-MB-231 cells, which are highly metastatic, though scratch wound healing and Matrigel invasion assays. It was suggested that this effect was due to impairments in the Rac1-WAVE2 pathway, as a reduction in Rac1 activity and also decreased expression of downstream effectors of Rac, such as WAVE2, Arp2, and Arp3, are seen. Further, Kim et al imply that treatment with rhapontigenin acts to inhibit invasive and migratory behavior in breast cancer cells through inhibition of formation of lamellipodia seen by observing morphological changes using immunofluorescence. ${ }^{81}$

\section{nWASP and WAVE2 in breast cancer cell invasion}

A study has observed three-dimensional invasion of MDAMB-231 cells that infiltrate through membrane pores in response to platelet-derived growth factor signals in a phosphatidylinositol-4,5-bisphosphate 3-kinase (PI3K)dependent manner, shown by using PI3K inhibitors. Immunofluorescence staining of these invading cells showed that WAVE2 and nWASP were colocalized with intensive F-actin accumulations at the site of infiltration. Further, diminution of WAVE2 and nWASP using siRNA techniques demonstrated a significant reduction in cell invasion, and accumulation of F-actin was also depleted. These findings suggest that nWASP and WAVE2 have a role in the threedimension invasion of MDA-MB-231 through small gaps in response to platelet-derived growth factor through reorganization of actin filaments within the cell. ${ }^{83}$ 


\section{WAVE3 and breast cancer}

Sossey-Alaoui et al identified WAVE3 as a candidate tumor suppressor gene on analysis of a patient with ganglioneuroblastoma in whom a chromosomal translocation causing a truncation of WAVE3 was seen. It was proposed that WAVE3 is important in cell differentiation and motility, considering it is part of the WASP/WAVE family that is involved in reorganization of the actin cytoskeleton, and that loss of WAVE3 activity seemed to be related to tumor formation in some forms of neuroblastoma ${ }^{84}$ Since this initial proposal, further work by Sossey-Alaoui et al has focused on elucidating the role of WAVE3 in cell motility and cancer metastasis.

It has been indicated that WAVE3 expression correlates with breast cancer progression, as immunohistochemical staining has shown significantly higher levels of WAVE3 in grade III tumors compared with low or no WAVE3 protein levels in normal breast or grade I tumors from human patients. ${ }^{85} \mathrm{~A}$ separate study has also identified WAVE3 activity as an indicator for some characteristics of breast cancer. WAVE3 expression levels were found to correlate with the invasiveness of MDA-MB-231 and BT-549 breast cancer cell lines compared with the less aggressive MCF7, T47D, and $\mathrm{SKBr} 3$ cell lines, shown in a Matrigel invasion assay. Immunohistochemistry and quantitative RT-PCR were also used to detect WAVE3 activity in tumor and blood samples, respectively, taken from breast cancer patients. The findings demonstrated that WAVE3 levels correlate with poor patient outcome in terms of incidence of metastatic disease, aggressiveness of subtype, and patient survival. ${ }^{86}$

Transient suppression of WAVE3 using siRNA techniques has been shown to impair the motility and invasiveness of MDA-MB-231 human breast cancer cells on Matrigel migration and gap closure assays. ${ }^{87}$ The same researchers also created a stable knockdown of WAVE3 protein expression in MDA-MB-231 cells using shRNA techniques. Suppression of WAVE3 activity was found to reduce the invasive ability of these cells by approximately fivefold on a Matrigel invasion assay. Further, knockdown of WAVE3 appeared to have an inhibitory effect on the metastatic potential of MDA-MB-231 cells. This was shown by the significantly reduced number of lung surface metastases seen after injection of the knockdown cells into the tail veins of SCID mice when compared with control cells. Finally, stable WAVE3 knockdown breast cancer cells were used in an orthotopic xenograft model where they were implanted into the mammary fat pads of female SCID mice. WAVE3-deficient cells were significantly less tumorigenic, with the tumor incidence reduced by $60 \%-80 \%$ compared with controls, and tumors that did develop grew more slowly and were less angiogenic. ${ }^{85}$ A more recent study has implicated WAVE3 in transforming growth factor- $\beta$-mediated epithelial to mesenchymal transition and metastasis of breast cancer cells. The highly metastatic murine triple-negative breast cancer cell model, 4T1, showed reduced levels of invasion in a Boyden chamber Matrigel assay when WAVE3 was inactivated through RNA silencing methods. Further, WAVE3 deficiency reduced the outgrowth of 4T1 organoids in three-dimensional cultures measured after 8 days and also reduced the growth and metastasis of triple-negative $4 \mathrm{~T} 1$ breast cancer cells engrafted into mammary glands or injected in the tails, respectively, of BALB/c mice. ${ }^{88}$

These studies further highlight the role of WAVE3 in the tumorigenic behavior of breast cancer cells and suggest that measures capable of inactivating WAVE3 may be able to act as a therapy targeted to breast cancer metastasis.

Interestingly, a study carried out by another group has disputed the finding by Sossey-Alaoui that WAVE3 is involved in the invasion of breast cancer cells in a Matrigel assay. ${ }^{90}$ Using RNA interference methods, Spence et al demonstrated that depletion of WAVE3 in MDA-MB-231 cells results in slower migration and larger and less dynamic lamellipodia when observed on a two-dimensional matrix. ${ }^{89}$ However, when invasion into Matrigel was observed in a three-dimensional environment, no difference was seen between WAVE3deficient cells and controls, so the group suggested that WAVE3 does not have a critical role in driving invasiveness of MDA-MB-231 cells in three-dimensional invasion assays. However, their results did show a preference of WAVE3deficient cells to invade in clusters of cells, which was speculated to indicate a loss of plasticity in WAVE3-deficient cells, thereby making them less able to change direction and break away from such clusters. No difference was found between WAVE3-deficient cells in MV3 and A375 metastatic melanoma cells. ${ }^{89}$ This study disagrees with the results of a similar study by Sossey-Alaoui et al, who reported that WAVE3 is important for breast cancer cell invasion through a Matrigel matrix. ${ }^{85}$ It has been suggested that the variation in outcomes observed in these similar studies could be due to differences in experimental procedures or perhaps the unknown use of a different cell line other than the intended MDA-MB-231 cells. ${ }^{89,90}$ Nonetheless, the involvement of WAVE3 in invasion and regulation of motility, and its metastatic potential has been demonstrated in numerous studies using several different cancer cell lines. ${ }^{68,70,85-88,91}$

It is clear that WAVE3 is a critical player in cancer metastasis and a biomarker for progression of breast cancer. 
More insights into the role of WAVE3 in the development of cancer are detailed in a comprehensive review by SosseyAlaoui, who has been involved in many of the advances in our understanding of the role of WAVE3. ${ }^{90}$

\section{Conclusion}

With the recognition that WASP/WAVE proteins are intrinsically involved in cell motility through regulation of actin polymerization, there has come the realization that this family could be involved in the motility of cancer cells and their invasive and metastatic potential. There have since been numerous investigations into the role of the WASP/WAVE family proteins in development of cancer. The evidence presented here confirms that WASP/WAVE proteins are linked to the migratory, invasive, and metastatic behavior of numerous cell types, including breast cancer cells, which have been the main focus of this review. A link between WASP/WAVE activity and the clinical outcome for breast cancer patients has also been described. Although recent studies have started to implicate the activators and pathways through which WASP/WAVEs may act to influence the behavior of cancer cells, further studies are required to fully elucidate which of the factors that regulate this family may be responsible for their role in cancer progression. Through doing so, we can target the WASP/WAVE family or the pathways that they are a part of in the hope of controlling the invasive and metastatic cell behavior that they have been implicated in. Many reports have highlighted the potential of targeting the WASP/WAVE family as a therapy for cancer progression, in particular breast cancer. It is important to determine the molecular mechanisms of WASP/WAVE activity and their exact contributions to the stages of cancer progression, thereby opening the door for development of anticancer drugs targeting the WASP/WAVE family and related pathways.

\section{Acknowledgments}

This work was supported by two sponsors, ie, the Life Science Research Network Wales, an initiative funded through Welsh Government's Sêr Cymru programme, and Cancer Research Wales.

\section{Disclosure}

The authors report that they have no conflicts of interest associated with this work.

\section{References}

1. Jemal A, Bray F, Center MM, Ferlay J, Ward E, Forman D. Global cancer statistics. CA Cancer J Clin. 2011;61:69-90.
2. Siegel R, Ma J, Zou Z, Jemal A. Cancer statistics. CA Cancer J Clin. 2014;64:9-29.

3. Steeg PS. Tumor metastasis: mechanistic insights and clinical challenges. Nat Med. 2006;12:895-904.

4. Ridley AJ, Schwartz MA, Burridge K, et al. Cell migration: integrating signals from front to back. Science. 2003;302:1704-1709.

5. Linder S, Wiesner C, Himmel M. Degrading devices: invadosomes in proteolytic cell invasion. Annu Rev Cell Dev Biol. 2011;27:185-211.

6. Ridley AJ. Life at the leading edge. Cell. 2011;145:1012-1022.

7. Insall RH, Machesky LM. Actin dynamics at the leading edge: from simple machinery to complex networks. Dev Cell. 2009;17:310-322.

8. Raftopoulou M, Hall A. Cell migration: Rho GTPases lead the way Dev Biol. 2004;265:23-32.

9. Takenawa T, Suetsugu S. The WASP-WAVE protein network: connecting the membrane to the cytoskeleton. Nat Rev Mol Cell Biol. 2007;8: $37-48$.

10. Yamaguchi H, Condeelis J. Regulation of the actin cytoskeleton in cancer cell migration and invasion. Biochim Biophys Acta. 2007;1773: 642-652.

11. Derry JM, Ochs HD, Francke U. Isolation of a novel gene mutated in Wiskott-Aldrich syndrome. Cell. 1994;78:635-644.

12. Aldrich RA, Steinberg AG, Campbell DC. Pedigree demonstrating a sexlinked recessive condition characterized by draining ears, eczematoid dermatitis and bloody diarrhea. Pediatrics. 1954;13:133-139.

13. Sullivan KE, Mullen CA, Blaese RM, Winkelstein JA. A multiinstitutional survey of the Wiskott-Aldrich syndrome. J Pediatr. 1994;125: 876-885.

14. Orange JS, Stone KD, Turvey SE, Krzewski K. The Wiskott-Aldrich syndrome. Cell Mol Life Sci. 2004;61:2361-2385.

15. Zhu Q, Watanabe C, Liu T, et al. Wiskott-Aldrich syndrome/X-linked thrombocytopenia: WASP gene mutations, protein expression, and phenotype. Blood. 1997;90:2680-2689.

16. Miki H, Miura K, Takenawa T. N-WASP, a novel actin-depolymerizing protein, regulates the cortical cytoskeletal rearrangement in a PIP2dependent manner downstream of tyrosine kinases. EMBOJ. 1996;15: 5326-5335.

17. Miki H, Suetsugu S, Takenawa T. WAVE, a novel WASP-family protein involved in actin reorganization induced by Rac. EMBO J. 1998; 17:6932-6941.

18. Machesky LM, Insall RH. Scar1 and the related Wiskott-Aldrich syndrome protein, WASP, regulate the actin cytoskeleton through the Arp2/3 complex. Curr Biol. 1998;8:1347-1356.

19. Suetsugu S, Miki H, Takenawa T. Identification of two human WAVE/ SCAR homologues as general actin regulatory molecules which associate with the Arp2/3 complex. Biochem Biophys Res Commun. 1999;260: 296-302.

20. Linardopoulou EV, Parghi SS, Friedman C, Osborn GE, Parkhurst SM, Trask BJ. Human subtelomeric WASH genes encode a new subclass of the WASP family. PLoS Genet. 2007;3:e237.

21. Campellone KG, Webb NJ, Znameroski EA, Welch MD. WHAMM is an Arp2/3 complex activator that binds microtubules and functions in ER to Golgi transport. Cell. 2008;134:148-161.

22. Zuchero JB, Coutts AS, Quinlan ME, Thangue NB, Mullins RD. p53cofactor JMY is a multifunctional actin nucleation factor. Nat Cell Biol. 2009;11:451-459.

23. Takenawa T, Miki H. WASP and WAVE family proteins. key molecules for rapid rearrangement of cortical actin filaments and cell movement. J Cell Sci. 2001;114:1801-1809.

24. Eden S, Rohatgi R, Podtelejnikov AV, Mann M, Kirschner MW. Mechanism of regulation of WAVE1-induced actin nucleation by Rac1 and Nck. Nature. 2002;418:790-793.

25. Gautreau A, Ho HY, Li J, Steen H, Gygi SP, Kirschner MW. Purification and architecture of the ubiquitous Wave complex. Proc Natl Acad Sci U S A. 2004;101:4379-4383.

26. Stovold CF, Millard TH, Machesky LM. Inclusion of Scar/WAVE3 in a similar complex to Scar/WAVE1 and 2. BMC Cell Biol. 2005;6:11. 
27. Innocenti M, Zucconi A, Disanza A, et al. Abil is essential for the formation and activation of a WAVE2 signalling complex. Nat Cell Biol. 2004;6:319-327.

28. Rohatgi R, Ma L, Miki H, et al. The interaction between N-WASP and the Arp2/3 complex links Cdc42-dependent signals to actin assembly. Cell. 1999;97:221-231.

29. Yarar D, To W, Abo A, Welch MD. The Wiskott-Aldrich syndrome protein directs actin-based motility by stimulating actin nucleation with the Arp2/3 complex. Curr Biol. 1999;9:555-558.

30. Kim AS, Kakalis LT, Abdul-Manan N, Liu GA, Rosen MK. Autoinhibition and activation mechanisms of the Wiskott-Aldrich syndrome protein. Nature. 2000;404:151-158.

31. Miki H, Sasaki T, Takai Y, Takenawa T. Induction of filopodium formation by a WASP-related actin-depolymerizing protein N-WASP. Nature. 1998;391:93-96.

32. Prehoda KE, Scott JA, Mullins RD, Lim WA. Integration of multiple signals through cooperative regulation of the N-WASP-Arp2/3 complex. Science. 2000;290:801-806.

33. Rohatgi R, Ho HY, Kirschner MW. Mechanism of N-WASP activation by CDC42 and phosphatidylinositol 4, 5-bisphosphate. J Cell Biol. 2000;150:1299-1310.

34. Derivery E, Lombard B, Loew D, Gautreau A. The Wave complex is intrinsically inactive. Cell Motil Cytoskeleton. 2009;66:777-790.

35. Ismail AM, Padrick SB, Chen B, Umetani J, Rosen MK. The WAVE regulatory complex is inhibited. Nat Struct Mol Biol. 2009;16:561-563.

36. Lebensohn AM, Kirschner MW. Activation of the WAVE complex by coincident signals controls actin assembly. Mol Cell. 2009;36: 512-524.

37. Oikawa T, Yamaguchi H, Itoh T, et al. PtdIns(3,4,5)P3 binding is necessary for WAVE2-induced formation of lamellipodia. Nat Cell Biol. 2004;6:420-426.

38. Suetsugu S, Kurisu S, Oikawa T, Yamazaki D, Oda A, Takenawa T. Optimization of WAVE2 complex-induced actin polymerization by membrane-bound IRSp53, PIP(3), and Rac. J Cell Biol. 2006;173: 571-585.

39. Derivery E, Gautreau A. Generation of branched actin networks: assembly and regulation of the N-WASP and WAVE molecular machines. Bioessays. 2010;32:119-131.

40. Euteneuer U, Schliwa M. Persistent, directional motility of cells and cytoplasmic fragments in the absence of microtubules. Nature. 1984;310:58-61.

41. Nemethova M, Auinger S, Small JV. Building the actin cytoskeleton: filopodia contribute to the construction of contractile bundles in the lamella. J Cell Biol. 2008;180:1233-1244.

42. Galbraith CG, Yamada KM, Galbraith JA. Polymerizing actin fibers position integrins primed to probe for adhesion sites. Science. 2007;315: 992-995.

43. Welch MD, Mullins RD. Cellular control of actin nucleation. Annu Rev Cell Dev Biol. 2002;18:247-288.

44. Chhabra ES, Higgs HN. The many faces of actin: matching assembly factors with cellular structures. Nat Cell Biol. 2007;9:1110-1121.

45. Lommel S, Benesch S, Rottner K, Franz T, Wehland J, Kuhn R. Actin pedestal formation by enteropathogenic Escherichia coli and intracellular motility of Shigella flexneri are abolished in N-WASP-defective cells. EMBO Rep. 2001;2:850-857.

46. Lorenz M, Yamaguchi H, Wang Y, Singer RH, Condeelis J. Imaging sites of N-wasp activity in lamellipodia and invadopodia of carcinoma cells. Curr Biol. 2004;14:697-703.

47. Kawamura K, Takano K, Suetsugu S, et al. N-WASP and WAVE2 acting downstream of phosphatidylinositol 3-kinase are required for myogenic cell migration induced by hepatocyte growth factor. $J$ Biol Chem. 2004;279:54862-54871.

48. Le Clainche C, Schlaepfer D, Ferrari A, et al. IQGAP1 stimulates actin assembly through the N-WASP-Arp2/3 pathway. $J$ Biol Chem. 2007;282:426-435.

49. Snapper SB, Takeshima F, Anton I, et al. N-WASP deficiency reveals distinct pathways for cell surface projections and microbial actin-based motility. Nat Cell Biol. 2001;3:897-904.
50. Innocenti $\mathrm{M}$, Gerboth $\mathrm{S}$, Rottner $\mathrm{K}$, et al. Abil regulates the activity of N-WASP and WAVE in distinct actin-based processes. Nat Cell Biol. 2005;7:969-976.

51. Rogers SL, Wiedemann U, Stuurman N, Vale RD. Molecular requirements for actin-based lamella formation in Drosophila S2 cells. J Cell Biol. 2003;162:1079-1088.

52. Suetsugu S, Yamazaki D, Kurisu S, Takenawa T. Differential roles of WAVE1 and WAVE2 in dorsal and peripheral ruffle formation for fibroblast cell migration. Dev Cell. 2003;5:595-609.

53. Krause M, Gautreau A. Steering cell migration: lamellipodium dynamics and the regulation of directional persistence. Nat Rev Mol Cell Biol. 2014; 15:577-590.

54. Yamazaki D, Suetsugu S, Miki H, et al. WAVE2 is required for directed cell migration and cardiovascular development. Nature. 2003;424: $452-456$.

55. Kurisu S, Suetsugu S, Yamazaki D, Yamaguchi H, Takenawa T. Rac-WAVE2 signaling is involved in the invasive and metastatic phenotypes of murine melanoma cells. Oncogene. 2005;24: 1309-1319.

56. Kheir WA, Gevrey JC, Yamaguchi H, Isaac B, Cox D. A WAVE2-Abi1 complex mediates CSF-1-induced F-actin-rich membrane protrusions and migration in macrophages. J Cell Sci. 2005;118:5369-5379.

57. Friedl $\mathrm{P}$, Wolf $\mathrm{K}$. Tumor-cell invasion and migration: diversity and escape mechanisms. Nat Rev Cancer. 2003;3:362-374.

58. Tang H, Li A, Bi J, et al. Loss of Scar/WAVE complex promotes N-WASP- and FAK-dependent invasion. Curr Biol. 2013;23:107-117.

59. Buccione R, Orth JD, McNiven MA. Foot and mouth: podosomes, invadopodia and circular dorsal ruffles. Nat Rev Mol Cell Biol. 2004;5: 647-657.

60. Yamaguchi H, Lorenz M, Kempiak S, et al. Molecular mechanisms of invadopodium formation: the role of the N-WASP-Arp2/3 complex pathway and cofilin. J Cell Biol. 2005;168:441-452.

61. Oser M, Yamaguchi H, Mader CC, et al. Cortactin regulates cofilin and N-WASp activities to control the stages of invadopodium assembly and maturation. J Cell Biol. 2009;186:571-587.

62. Mizutani K, Miki H, He H, Maruta H, Takenawa T. Essential role of neural Wiskott-Aldrich syndrome protein in podosome formation and degradation of extracellular matrix in src-transformed fibroblasts. Cancer Res. 2002;62:669-674.

63. Gligorijevic B, Wyckoff J, Yamaguchi H, Wang Y, Roussos ET, Condeelis J. N-WASP-mediated invadopodium formation is involved in intravasation and lung metastasis of mammary tumors. J Cell Sci. 2012;125:724-734.

64. Coopman PJ, Do MT, Thompson EW, Mueller SC. Phagocytosis of cross-linked gelatin matrix by human breast carcinoma cells correlates with their invasive capacity. Clin Cancer Res. 1998;4:507-515.

65. Yang LY, Tao YM, Ou DP, Wang W, Chang ZG, Wu F. Increased expression of Wiskott-Aldrich syndrome protein family verprolin-homologous protein 2 correlated with poor prognosis of hepatocellular carcinoma. Clin Cancer Res. 2006;12:5673-5679.

66. Semba S, Iwaya K, Matsubayashi J, et al. Coexpression of actinrelated protein 2 and Wiskott-Aldrich syndrome family verprolinehomologous protein 2 in adenocarcinoma of the lung. Clin Cancer Res. 2006;12:2449-2454.

67. Iwaya K, Oikawa K, Semba S, et al. Correlation between liver metastasis of the colocalization of actin-related protein 2 and 3 complex and WAVE2 in colorectal carcinoma. Cancer Sci. 2007;98: 992-999.

68. Fernando HS, Sanders AJ, Kynaston HG, Jiang WG. WAVE3 is associated with invasiveness in prostate cancer cells. Urol Oncol. 2010;28: 320-327.

69. Fernando HS, Sanders AJ, Kynaston HG, Jiang WG. WAVE1 is associated with invasiveness and growth of prostate cancer cells. J Urol. 2008;180:1515-1521.

70. Teng Y, Ren MQ, Cheney R, Sharma S, Cowell JK. Inactivation of the WASF3 gene in prostate cancer cells leads to suppression of tumorigenicity and metastases. Br J Cancer. 2010;103:1066-1075. 
71. Ishihara D, Dovas A, Hernandez L, et al. Wiskott-Aldrich syndrome protein regulates leukocyte-dependent breast cancer metastasis. Cell Rep. 2013;4:429-436.

72. Goswami S, Sahai E, Wyckoff JB, et al. Macrophages promote the invasion of breast carcinoma cells via a colony-stimulating factor-1/epidermal growth factor paracrine loop. Cancer Res. 2005;65: 5278-5283.

73. Wyckoff J, Wang W, Lin EY, et al. A paracrine loop between tumor cells and macrophages is required for tumor cell migration in mammary tumors. Cancer Res. 2004;64:7022-7029.

74. Zicha D, Allen WE, Brickell PM, et al. Chemotaxis of macrophages is abolished in the Wiskott-Aldrich syndrome. Br J Haematol. 1998;101: 659-665.

75. Escudero-Esparza A, Jiang WG, Martin TA. Claudin-5 is involved in breast cancer cell motility through the N-WASP and ROCK signalling pathways. J Exp Clin Cancer Res. 2012;31:43.

76. Peterson JR, Bickford LC, Morgan D, et al. Chemical inhibition of N-WASP by stabilization of a native autoinhibited conformation. Nat Struct Mol Biol. 2004;11:747-755.

77. Martin TA, Pereira G, Watkins G, Mansel RE, Jiang WG. N-WASP is a putative tumor suppressor in breast cancer cells, in vitro and in vivo, and is associated with clinical outcome in patients with breast cancer. Clin Exp Metastasis. 2008;25:97-108.

78. Pichot CS, Arvanitis C, Hartig SM, et al. Cdc42-interacting protein 4 promotes breast cancer cell invasion and formation of invadopodia through activation of N-WASp. Cancer Res. 2010;70: 8347-8356.

79. Sanchez AM, Flamini MI, Baldacci C, Goglia L, Genazzani AR, Simoncini T. Estrogen receptor-alpha promotes breast cancer cell motility and invasion via focal adhesion kinase and N-WASP. Mol Endocrinol. 2010;24:2114-2125.

80. Fernando HS, Davies SR, Chhabra A, et al. Expression of the WASP verprolin-homologues (WAVE members) in human breast cancer. Oncology. 2007;73:376-383.

81. Kim JS, Kang CG, Kim SH, Lee EO. Rhapontigenin suppresses cell migration and invasion by inhibiting the PI3K-dependent Rac1 signaling pathway in MDA-MB-231 human breast cancer cells. J Nat Prod. 2014;77:1135-1139
82. Ko HS, Kim JS, Cho SM, et al. Urokinase-type plasminogen activator expression and Rac1/WAVE-2/Arp2/3 pathway are blocked by pterostilbene to suppress cell migration and invasion in MDA-MB-231 cells. Bioorg Med Chem Lett. 2014;24:1176-1179.

83. Takahashi K, Suzuki K. WAVE2, N-WASP, and Mena facilitate cell invasion via phosphatidylinositol 3-kinase-dependent local accumulation of actin filaments. J Cell Biochem. 2011;112:3421-3429.

84. Sossey-Alaoui K, Su G, Malaj E, Roe B, Cowell JK. WAVE3, an actin-polymerization gene, is truncated and inactivated as a result of a constitutional $\mathrm{t}(1 ; 13)(\mathrm{q} 21 ; \mathrm{q} 12)$ chromosome translocation in a patient with ganglioneuroblastoma. Oncogene. 2002;21:5967-5974.

85. Sossey-Alaoui K, Safina A, Li X, et al. Down-regulation of WAVE3, a metastasis promoter gene, inhibits invasion and metastasis of breast cancer cells. Am J Pathol. 2007;170:2112-2121.

86. Kulkarni S, Augoff K, Rivera L, et al. Increased expression levels of WAVE3 are associated with the progression and metastasis of triple negative breast cancer. PLoS One. 2012;7:e42895.

87. Sossey-Alaoui K, Ranalli TA, Li X, Bakin AV, Cowell JK. WAVE3 promotes cell motility and invasion through the regulation of MMP-1, MMP-3, and MMP-9 expression. Exp Cell Res. 2005;308:135-145.

88. Taylor MA, Davuluri G, Parvani JG, et al. Upregulated WAVE3 expression is essential for TGF-beta-mediated EMT and metastasis of triple-negative breast cancer cells. Breast Cancer Res Treat. 2013;142: 341-353.

89. Spence HJ, Timpson P, Tang HR, Insall RH, Machesky LM. Scar/ WAVE3 contributes to motility and plasticity of lamellipodial dynamics but not invasion in three dimensions. Biochem J. 2012;448:35-42.

90. Sossey-Alaoui K. Surfing the big WAVE: insights into the role of WAVE3 as a driving force in cancer progression and metastasis. Semin Cell Dev Biol. 2013;24:287-297.

91. Teng Y, Ngoka L, Mei Y, Lesoon L, Cowell JK. HSP90 and HSP70 proteins are essential for stabilization and activation of WASF3 metastasispromoting protein. J Biol Chem. 2012;287:10051-10059.
Breast Cancer: Targets and Therapy

\section{Publish your work in this journal}

Breast Cancer: Targets and Therapy is an international, peerreviewed open access journal focusing on breast cancer research, identification of therapeutic targets and the optimal use of preventative and integrated treatment interventions to achieve improved outcomes, enhanced survival and quality of life for the cancer patient.

\section{Dovepress}

View the full aims and scopes of this journal here. The manuscript management system is completely online and includes a very quick and fair peer-review system, which is all easy to use. Visit http:// www.dovepress.com/testimonials.php to read real quotes from published authors. 